МИНИСТЕРСТВО ОБРАЗОВАНИЯ И НАУКИ РОССИЙСКОЙ ФЕДЕРАЦИИ

ТОМСКИЙ ГОСУДАРСТВЕННЫЙ УНИВЕРСИТЕТ

\title{
МАТЕРИАЛЫ
}

VIII Международной молодежной научной конференции

«МАТЕМАТИЧЕСКОЕ

И ПРОГРАММНОЕ ОБЕСПЕЧЕНИЕ

ИНФОРМАЦИОННЫХ, ТЕХНИЧЕСКИХ

И ЭКОНОМИЧЕСКИХ СИСТЕМ»

Томск, 26-30 мая 2021 г.

Под общей редакиией И.С. Шмырина

Томск

Издательство Томского государственного университета 2021 


\title{
IV. ОБРАБОТКА ДАННЫХ, УПРАВЛЕНИЕ И ИССЛЕДОВАНИЕ СТОХАСТИЧЕСКИХ СИСТЕМ
}

DOI: $10.17223 / 978-5-907442-42-9-2021-12$

\section{ОЦЕНИВАНИЕ МЕТОДОМ МОМЕНТОВ ПАРАМЕТРА \\ РАВНОМЕРНОГО РАСПРЕДЕЛЕНИЯ ДЛИТЕЛЬНОСТИ \\ СЛУЧАЙНОГО НЕПРОДЛЕВАЮЩЕГОСЯ МЕРТВОГО ВРЕМЕНИ В РЕКУРРЕНТНОМ ПОЛУСИНХРОННОМ ПОТОКЕ СОБЫТИЙ}

\author{
Горцев А.М., Веткина А.В. \\ Томский государственный университет \\ anyavetkina@stud.tsu.ru
}

Введение

В настоящее время наиболее точной и адекватной математической моделью реальных информационных потоков в телекоммуникационных системах и сетях являются дважды стохастические потоки событий - это потоки, у которых случайными являются и моменты наступления событий, и интенсивность потока. В связи с этим исследование разновидностей дважды стохастических потоков становится важной актуальной задачей.

Дважды стохастические потоки можно разделить на два класса: первый класс составляют потоки, сопровождающий процесс которых есть непрерывный случайный процесс [1,2]; второй - потоки, сопровождающий процесс которых есть кусочнопостоянный случайный процесс с конечным (произвольным) числом состояний [3-5].

Помимо этого, в зависимости от того, каким образом происходит переход сопровождающего процесса из состояния в состояние, дважды стохастические потоки делятся на три типа: 1) синхронные потоки (потоки, у которых состояние сопровождающего процесса меняется в случайные моменты времени, являющиеся моментами наступления событий) [6-9]; 2) асинхронные потоки (потоки, у которых переход из состояния в состояние сопровождающего процесса происходит в случайные моменты времени и не зависит от моментов наступления событий) [10-13]; 3) полусинхронные потоки (потоки, у которых одна часть состояний сопровождающего процесса меняется в моменты наступления событий потока, другая часть состояний сопровождающего процесса меняется в произвольные моменты времени, не связанные с моментами наступления событий потока) [14-17].

На практике часто приходится иметь дело с потоками, у которых не все события доступны наблюдению. Как правило, причиной ненаблюдаемости служит так называемое мертвое время регистрирующих приборов [18], порождаемое зарегистрированным событием, другие же события, наступившие в этот период, теряются. Регистрирующие приборы, вместе с тем, делятся на два вида: с непродлевающимся мертвым временем и продлевающимся. Кроме того, длительность мертвого времени может быть как детерминированной величиной, одинаковой для всех событий, так и случайной с тем или иным законом распределения [18].

В реальности, наблюдая за системами различного вида, исследователь имеет лишь информацию на выходе (случайную выборку данных), а то, что подается на вход системы, остается для него неизвестным. Именно поэтому важной задачей становится задача оценивания входных параметров потока с помощью использования наблюденных данных.

В данной работе рассматривается полусинхронный дважды стохастический поток событий, функционирующий в условиях случайного непродлевающегося мертвого 
времени, распределенного по равномерному закону. Выводится явный вид плотности вероятности значений длительности интервала между моментами наступления событий наблюдаемого потока, а также находится явный вид совместной плотности вероятности значений длительностей смежных интервалов. Находятся условия рекуррентности потока в рассматриваемом случае. Для рекуррентного потока методом моментов решается задача оценивания параметра распределения длительности случайного мертвого времени.

\section{1. Математическая модель наблюдаемого потока}

Рассматривается стационарный режим функционирования полусинхронного дважды стохастического потока событий, сопровождающий процесс (интенсивность) которого есть кусочно-постоянный стационарный случайный процесс $\lambda(t)$ с двумя состояниями $S_{1}$ и $S_{2}$. Будем говорить, что имеет место первое состояние процесса (потока) $S_{1}$, если $\lambda(t)=\lambda_{1}$ и, наоборот, имеет место второе состояние процесса (потока) $S_{2}$, если $\lambda(t)=\lambda_{2}\left(\lambda_{1}>\lambda_{2} \geq 0\right)$. Если имеет место первое состояние процесса $S_{1}$, то в течение временного интервала, когда $\lambda(t)=\lambda_{1}$, поступает пуассоновский поток событий с интенсивностью $\lambda_{1}$. Если имеет место второе состояние процесса $S_{2}$, то в течение временного интервала, когда $\lambda(t)=\lambda_{2}$, поступает пуассоновский поток событий с интенсивностью $\lambda_{2}$. Переход из состояния $S_{1}$ процесса $\lambda(t)$ в состояние $S_{2}$ возможен только в момент наступления события, при этом этот переход осуществляется с вероятностью $p$ (с вероятностью $1-p$ процесс $\lambda(t)$ остается в состоянии $S_{1}$ ). Переход из состояния $S_{2}$ процесса $\lambda(t)$ в состояние $S_{1}$ может осуществляться в произвольный момент времени, не связанный с моментом наступления события. При этом длительность пребывания процесса $\lambda(t)$ во втором состоянии есть случайная величина, распределенная по экспоненциальному закону $F(t)=1-e^{-\alpha_{2} t}, t \geq 0$, где $\alpha_{2}-$ интенсивность смены состояния $S_{2}$ на $S_{1}$. Так как переход из второго состояния в первое не привязан к моменту наступления события во втором состоянии, то поток называется полусинхронным дважды стохастическим потоком событий. В сделанных предположениях $\lambda(\mathrm{t})-$ скрытый марковский процесс $(\lambda(t)$ - принципиально ненаблюдаемый процесс; наблюдаемыми являются только моменты наступления событий потока).

После каждого зарегистрированного события в момент времени $t_{k}$ наступает период мертвого времени случайной длительности, который порождается этим событием, так что другие события исходного потока, наступившие в течение этого периода мертвого времени, недоступны наблюдению и не вызывают его продления (непродлевающееся мертвое время). Принимается, что случайная длительность мертвого времени распределена по равномерному закону с плотностью вероятности $p(T)=1 / T^{*}$, где $T-$ значение длительности мертвого времени, $0 \leq T \leq T^{*}$.

Возможный вариант возникающей ситуации приведен на рис. 1 , где $S_{1}$ и $S_{2}$ - состояния случайного процесса $\lambda(t)$; временная ось $(0, t)$ - ось моментов наступления наблюдаемых событий в моменты времени $t_{1}, t_{2}, \ldots$; временная ось $\left(0, t^{(1)}\right)$ - ось наступления событий в моменты времени $t^{(1)}, t^{(2)}, \ldots$ в первом $\left(S_{1}\right)$ состоянии процесса $\lambda(t)$, на которой также указаны значения длительностей $T^{(1)}, T^{(2)}, \ldots$ мертвых времен, порождаемых наблюдаемыми событиями потока; аналогично для временной оси $\left(0, t^{(2)}\right)$; белыми кружками обозначены наблюдаемые события, черными - ненаблюдаемые, штриховкой - периоды продолжительности мертвого времени; траектория процесса $\lambda(t)$ привязана к временной оси $\left(0, t^{(1)}\right)$. 


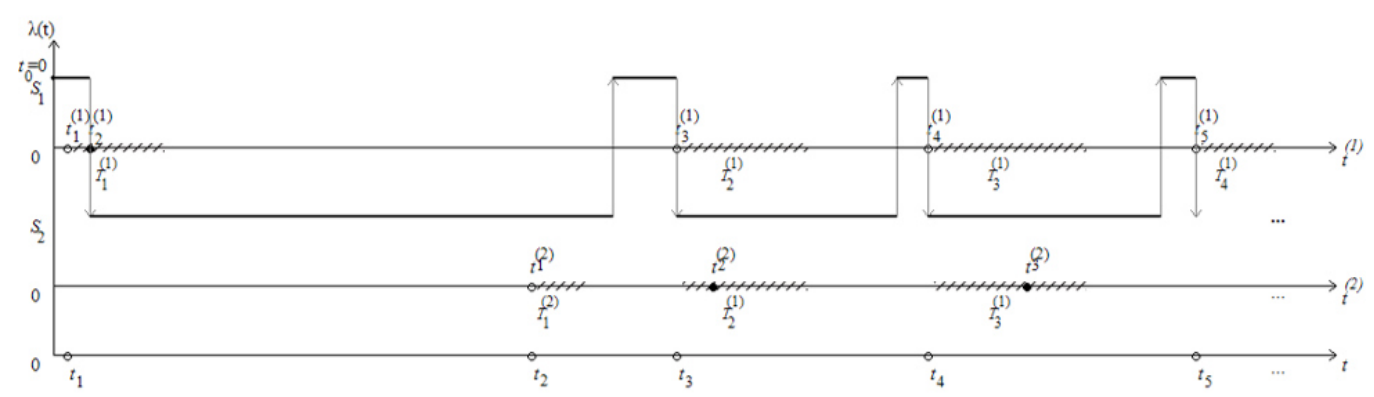

Рис. 1. Формирование наблюдаемого полусинхронного потока событий

Рассмотрим теперь полусинхронный поток событий, функционирующий в условиях непродлевающегося мертвого времени фиксированной длительности $T \geq 0$; если $T=0$, то мертвое время отсутствует. Из [17] имеем, что плотность вероятности $p(\tau \mid T)$ значений длительности интервала между соседними событиями в полусинхронном потоке, функционирующем в условиях непродлевающегося мертвого времени фиксированной длительности $T$ имеет вид

$$
\begin{gathered}
p(\tau \mid T)=\left\{\begin{array}{l}
0,0 \leq \tau<T, \\
\gamma(T) \lambda_{1} e^{-\lambda_{1}(\tau-T)}+(1-\gamma(T))\left(\alpha_{2}+\lambda_{2}\right) e^{-\left(\alpha_{2}+\lambda_{2}\right)(\tau-T)}, \tau \geq T,
\end{array}\right. \\
p\left(\tau_{1}, \tau_{2} \mid T\right)=0, \tau_{2} \geq 0,0 \leq \tau_{1}<T, \quad \tau_{1} \geq 0, \quad 0 \leq \tau_{2}<T, \\
p\left(\tau_{1}, \tau_{2} \mid T\right)=p\left(\tau_{1} \mid T\right) p\left(\tau_{2} \mid T\right)+e^{-\left(\alpha_{2}+p \lambda_{1}\right) T} \gamma(T)[1-\gamma(T)] \frac{\lambda_{2}(1-p)}{\lambda_{2}+\alpha_{2}} \times \\
\times\left[\lambda_{1} e^{-\lambda_{1}\left(\tau_{1}-T\right)}-\left(\lambda_{2}+\alpha_{2}\right) e^{-\left(\lambda_{2}+\alpha_{2}\right)\left(\tau_{1}-T\right)}\right]\left[\lambda_{1} e^{-\lambda_{1}\left(\tau_{2}-T\right)}-\left(\lambda_{2}+\alpha_{2}\right) e^{-\left(\lambda_{2}+\alpha_{2}\right)\left(\tau_{2}-T\right)}\right], \\
\tau_{1} \geq T, \quad \tau_{2} \geq T,
\end{gathered}
$$

где $\gamma(T)=\frac{\alpha_{2}\left(\lambda_{1}-\lambda_{2}-\alpha_{2}-\lambda_{1} p\right)}{\left(\lambda_{1} p+\alpha_{2}\right)\left(\lambda_{1}-\lambda_{2}-\alpha_{2}\right)}\left[1-\frac{p\left(\lambda_{1}-\lambda_{2}\right)}{(1-p) \lambda_{2}-\left(\alpha_{2}+\lambda_{2}\right) e^{\left(\lambda_{1} p+\alpha_{2}\right) T}}\right], \lambda_{1}-\lambda_{2}-\alpha_{2} \neq 0 ; \tau_{1}$, $\tau_{2}$ - смежные интервалы между наблюдаемыми событиями.

Рассмотрим частные случаи, при выполнении которых полусинхронный поток событий, функционирующий в условиях мертвого времени, становится рекуррентным потоком:

1) $p=0 ;$ тогда $\gamma(T)=1$ и совместная плотность (2) факторизуется $p\left(\tau_{1}, \tau_{2} \mid T\right)=p\left(\tau_{1} \mid T\right) p\left(\tau_{2} \mid T\right), \tau_{1} \geq T, \tau_{2} \geq T$, а плотность вероятности (1) принимает вид $p(\tau \mid T)=\lambda_{1} e^{-\lambda_{1}(\tau-T)}, \tau \geq T$; в этом случае имеет место простейший поток с интенсивностью $\lambda_{1}$.

2) $\alpha_{2}=0 ; \quad$ тогда $\quad \gamma(T)=0, \quad p\left(\tau_{1}, \tau_{2} \mid T\right)=p\left(\tau_{1} \mid T\right) p\left(\tau_{2} \mid T\right), \quad \tau_{1} \geq T, \quad \tau_{2} \geq T$, $p(\tau \mid T)=\lambda_{2} e^{-\lambda_{2}(\tau-T)}, \tau \geq T$; в этом случае имеет место простейший поток с интенсивностью $\lambda_{2}$.

3) $\lambda_{1}(1-p)-\lambda_{2}-\alpha_{2}=0$; тогда $\gamma(T)=0, \quad p\left(\tau_{1}, \tau_{2} \mid T\right)=p\left(\tau_{1} \mid T\right) p\left(\tau_{2} \mid T\right), \quad \tau_{1} \geq T$, $\tau_{2} \geq T, p(\tau \mid T)=\left(\lambda_{2}+\alpha_{2}\right) e^{-\left(\lambda_{2}+\alpha_{2}\right)(\tau-T)}, \tau \geq T$; в этом случае имеет место простейший поток с интенсивностью $\lambda_{2}+\alpha_{2}$.

4) $\lambda_{2}=0$; в данной ситуации имеет место 


$$
\begin{gathered}
p\left(\tau_{1}, \tau_{2} \mid T\right)=p\left(\tau_{1} \mid T\right) p\left(\tau_{2} \mid T\right), \tau_{2} \geq 0,0 \leq \tau_{1}<T, \tau_{1} \geq 0,0 \leq \tau_{2}<T, \\
\gamma(T)=\frac{\alpha_{2}\left(\lambda_{1}-\alpha_{2}-\lambda_{1} p\right)}{\left(\lambda_{1} p+\alpha_{2}\right)\left(\lambda_{1}-\alpha_{2}\right)}\left[1+\frac{p \lambda_{1}}{\alpha_{2} e^{\left(\lambda_{1} p+\alpha_{2}\right) T}}\right], \\
p(\tau \mid T)=\gamma(T) \lambda_{1} e^{-\lambda_{1}(\tau-T)}+(1-\gamma(T))\left(\alpha_{2}+\lambda_{2}\right) e^{-\left(\alpha_{2}+\lambda_{2}\right)(\tau-T)}, \tau \geq T, \lambda_{1}-\alpha_{2} \neq 0 .
\end{gathered}
$$

Формулы (3) характеризуют альтернирующий рекуррентный полусинхронный поток (отсутствуют события во втором состоянии).

Рассмотрим четвертый случай альтернирующего рекуррентного полусинхронного потока событий, когда $\lambda_{2}=0$. На рис. 2 приведен вариант формирования такого наблюдаемого потока при фиксированном $T>0$.

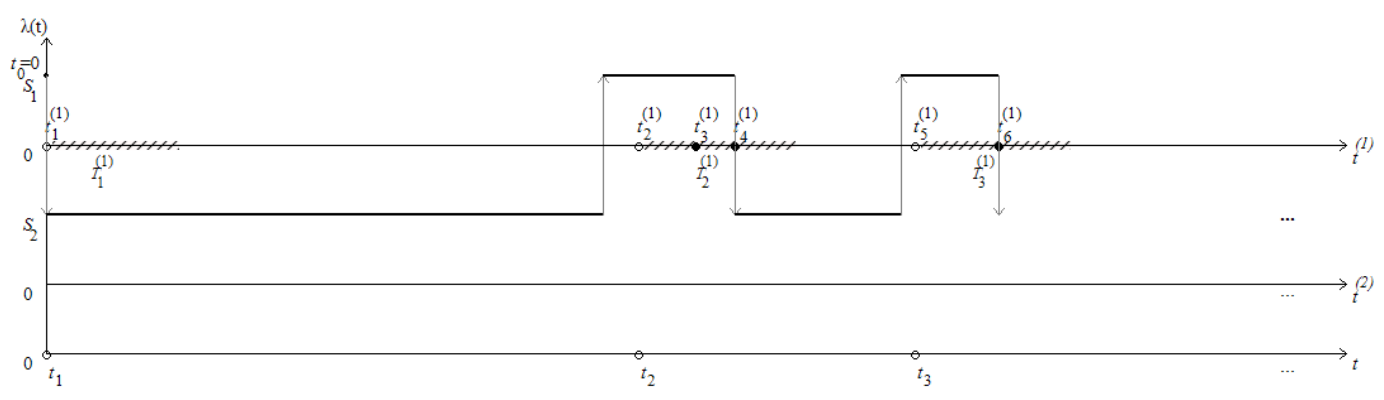

Рис. 2. Формирование наблюдаемого альтернирующего рекуррентного полусинхронного потока событий

\section{2. Уравнение моментов для оценивания параметра $T^{*}$}

Предварительно отметим, что условия рекуррентности потока 1) - 4), полученные для случая детерминированного мертвого времени, для ситуации случайного непродлевающегося мертвого времени, очевидно, не изменятся, т.к. они являются следствием значений параметров потока или их соотношений. Условия 1) - 3) рекуррентности потока приводят к тому, что исходный исследуемый поток вырождается в простейший, поэтому рассмотрим четвертое условия рекуррентности.

Для оценки неизвестного параметра $T^{*}$ равномерного распределения длительности случайного непродлевающегося мертвого времени в рекуррентном полусинхронном потоке событий, когда $\lambda_{2}=0$, используется метод моментов [19]. Метод моментов заключается в приравнивании теоретических и эмпирических моментов. Для этого находится теоретический момент - математическое ожидание длительности интервала между соседними событиями в наблюдаемом потоке $M\left(\tau \mid T^{*}\right)$, а затем значение оценки $\hat{\boldsymbol{T}}^{*}$ вычисляется численно из уравнения моментов $M\left(\tau \mid T^{*}\right)=C$, где $C$ - значение оценки математического ожидания $M\left(\tau \mid T^{*}\right), C=\frac{1}{n} \sum_{k=1}^{n} \tau_{k}, \tau_{k}=t_{k+1}-t_{k}$. Величины $\tau_{k}$ являются выходными данными имитационной модели наблюдаемого альтернирующего рекуррентного полусинхронного потока событий.

Для нахождения теоретического момента имеем формулу $M\left(\tau \mid T^{*}\right)=\int_{0}^{\infty} p(\tau) d \tau$. Здесь плотность вероятности $p(\tau)=\int_{(T)} p(\tau, T) d T=\int_{(T)} p(T) p(\tau \mid T) d T$, где $(T)$ - область изменения значений случайной величины $T ; p(T)=1 / T^{*}, 0 \leq T \leq T^{*}$, по условию задачи. 
Так как область значений случайной величины мертвого времени представляет собой объединение двух областей, когда $0 \leq \tau<T^{*}$ и когда $\tau \geq T^{*}$, то выражение для плотности $p(\tau)$ примет следующий вид:

$$
p(\tau)= \begin{cases}p_{1}(\tau)=\int_{0}^{\tau} p(T) p(\tau \mid T) d T, & 0 \leq \tau<T^{*}, \\ p_{2}(\tau)=\int_{0}^{T^{*}} p(T) p(\tau \mid T) d T, & \tau \geq T^{*},\end{cases}
$$

где плотность $p(\tau \mid T)$ определена в (3).

Подставляя выражение (3) в (4) и учитывая, что $p(T)=1 / T^{*}$, находим

$$
\begin{gathered}
p_{1}(\tau)=\frac{1}{T^{*}}\left\{1-e^{-\lambda_{1} \tau}-e^{-\alpha_{2} \tau}+e^{-\left(\lambda_{1} p+\alpha_{2}\right) \tau}\right\}, 0 \leq \tau<T^{*} \\
p_{2}(\tau)=\frac{1}{T^{*}}\left\{-e^{-\lambda_{1} \tau}\left[-1+C_{1} e^{\lambda_{1} T^{*}}+C_{2} e^{\left(\lambda_{1}-\lambda_{1} p-\alpha_{2}\right) T^{*}}\right]+e^{-\alpha_{2} \tau}\left[-1+C_{1} e^{-\lambda_{1} p T^{*}}+C_{2} e^{\alpha_{2} T^{*}}\right]\right\}, \tau \geq T^{*},(6) \\
\text { где } C_{1}=\frac{\alpha_{2}\left(\lambda_{1}-\alpha_{2}-\lambda_{1} p\right)}{\left(\lambda_{1} p+\alpha_{2}\right)\left(\lambda_{1}-\alpha_{2}\right)}, C_{2}=\frac{\lambda_{1}^{2} p}{\left(\lambda_{1} p+\alpha_{2}\right)\left(\lambda_{1}-\alpha_{2}\right)} .
\end{gathered}
$$

Отметим, что в граничной точке $\tau=T^{*}$ имеет место равенство двух плотностей $p_{1}\left(T^{*}\right)=p_{2}\left(T^{*}\right)$ и неравенство их производных $p_{1}^{\prime}\left(T^{*}\right) \neq p_{2}^{\prime}\left(T^{*}\right)$, т.е. функция $p(\tau)$, задаваемая формулой (4), есть непрерывная функция и в точке $\tau=T^{*}$ имеет излом.

По определению математического ожидания случайной величины $\boldsymbol{\tau}$ - длительности интервала между двумя соседними событиями наблюдаемого потока - с учетом формулы (4), имеем $M\left(\tau \mid T^{*}\right)=\int_{0}^{T^{*}} \tau p_{1}(\tau) d \tau+\int_{T^{*}}^{\infty} \tau p_{2}(\tau) d \tau$. Подставляя сюда плотности вероятности $p_{1}(\tau), p_{2}(\tau)$, определенные в (5), (6), находим

$$
M\left(\tau \mid T^{*}\right)=\frac{T^{*}}{2}+\frac{1}{\lambda_{1}}+\frac{\lambda_{1} p}{\alpha_{2}\left(\lambda_{1} p+\alpha_{2}\right)}+\frac{p\left(\lambda_{1}(1-p) p+\alpha_{2}\right)}{T^{*} \alpha_{2}\left(\lambda_{1} p+\alpha_{2}\right)^{2}}\left(1-e^{-\left(\lambda_{1} p+\alpha_{2}\right) T^{*}}\right), T^{*} \geq 0 .
$$

Аналитически можно показать, что $M^{\prime}\left(\tau \mid T^{*}\right)>0$ при $T^{*}>0 ; \lim M^{\prime}\left(\tau \mid T^{*}\right)>0$ при $T^{*} \rightarrow 0$. Это означает, что функция $M\left(\tau \mid T^{*}\right)$ является возрастающей функцией $T^{*}$.

Теперь для нахождения значения оценки $\hat{\boldsymbol{T}}^{*}$ необходимо решить уравнение моментов $M\left(\tau \mid T^{*}\right)=C$. Данное уравнение будем решать численно методом простой итерации [20].

Отметим, что уравнение моментов $M\left(\tau \mid T^{*}\right)=C$ имеет единственное решение, т.к. можно показать, что математическое ожидание $M\left(\tau \mid T^{*}\right)$ - возрастающая функция переменной $T^{*}, C$ - постоянная величина, характеризующая конкретную реализацию потока.

\section{3. Численные результаты}

С целью установления стационарного режима и определения свойств найденной оценки проведены статистические эксперименты. Для параметров потока $\lambda_{1}=2$, $\lambda_{2}=0, \alpha_{2}=0,2, \mathrm{p}=0,6$ и параметров точности $\varepsilon=0.0001, \Delta T^{*}=0.001$ (параметры метода простой итерации) получено 100 реализаций $(N=100)$ имитационной модели 
потока и, соответственно, получено 100 решений уравнения моментов, для двух значений параметра $T^{*}=1 ; 3$ и для каждого значения времени моделирования $T_{m}=150,300, \ldots, 3000$ ед. времени. Далее на основании полученных данных вычислялись выборочное среднее искомой оценки $\hat{M}\left(\hat{\boldsymbol{T}}^{*}\right)=\frac{1}{N} \sum_{i=1}^{N} \hat{T}_{i}^{*}$ и ее выборочная вариация $\operatorname{Var}\left(\hat{\boldsymbol{T}}^{*}\right)=\frac{1}{N} \sum_{i=1}^{N}\left(\hat{T}_{i}^{*}-T^{*}\right)^{2}$, где $T^{*}-$ известное из имитационной модели значение параметра, $\hat{T}_{i}^{*}$ - решение уравнения моментов для $i$-й реализации потока.

В табл. 1 приведены результаты эксперимента для $\hat{M}\left(\hat{\boldsymbol{T}}^{*}\right)$. В первой строке таблицы указано время моделирования $T_{m}$ (время наблюдения за потоком) $\left(T_{m}=150,300, \ldots, 3000\right.$ ед. времени); во второй и третьей строках указано выборочное среднее $\hat{M}\left(\hat{\boldsymbol{T}}^{*}\right)$ для $T^{*}=1$ и $T^{*}=3$ соответственно.

Таблица 1

Численные результаты статистического эксперимента для $\hat{\boldsymbol{M}}\left(\hat{\boldsymbol{T}}^{*}\right)$

\begin{tabular}{|c|c|l|l|l|l|l|l|l|}
\hline $\boldsymbol{T}_{\boldsymbol{m}}$ & & $\mathbf{1 5 0}$ & $\mathbf{3 0 0}$ & $\mathbf{4 5 0}$ & $\mathbf{6 0 0}$ & $\mathbf{7 5 0}$ & $\mathbf{9 0 0}$ & $\mathbf{1 0 5 0}$ \\
\hline \multirow{2}{*}{$\hat{\boldsymbol{M}}\left(\hat{\boldsymbol{T}}^{*}\right)$} & $\boldsymbol{T}^{*}=\mathbf{1}$ & 1.31445 & 1.03263 & 1.02797 & 1.08673 & 1.02456 & 1.07423 & 0.99706 \\
\cline { 2 - 8 } & $\boldsymbol{T}^{*}=\mathbf{3}$ & 3.35504 & 2.91497 & 2.89639 & 3.00945 & 2.96404 & 2.9861 & 3.04309 \\
\hline $\boldsymbol{T}_{\boldsymbol{m}}$ & $\mathbf{1 2 0 0}$ & $\mathbf{1 3 5 0}$ & $\mathbf{1 5 0 0}$ & $\mathbf{1 6 5 0}$ & $\mathbf{1 8 0 0}$ & $\mathbf{1 9 5 0}$ & $\mathbf{2 1 0 0}$ & $\mathbf{2 2 5 0}$ \\
\hline \multirow{2}{*}{$\left(\hat{\boldsymbol{T}}^{*}\right)$} & 1.0058 & 1.02834 & 0.98195 & 1.0389 & 0.99737 & 1.01769 & 1.00434 & 1.01259 \\
\cline { 2 - 8 } & 2.9291 & 2.9892 & 2.98173 & 3.00373 & 2.95898 & 2.98324 & 3.04984 & 2.98432 \\
\hline
\end{tabular}

Для наглядности на рис. 3, 4 приведены графики зависимости $\hat{M}\left(\hat{\boldsymbol{T}}^{*}\right)$ от значения времени моделирования $T_{m}$ для $T^{*}=1$ и $T^{*}=3$, построенные по данным табл. 1 .

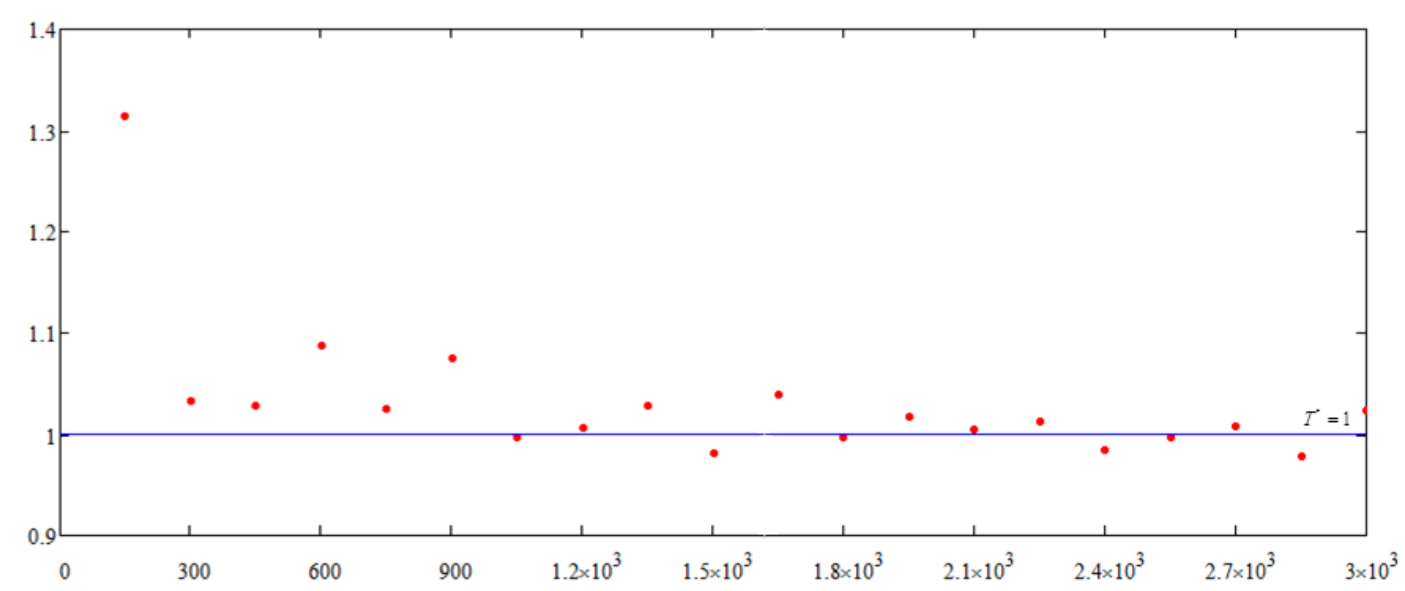

Рис. 3. График зависимости $\hat{M}\left(\hat{\boldsymbol{T}}^{*}\right)$ от $T_{m}$ при $T^{*}=1$ 


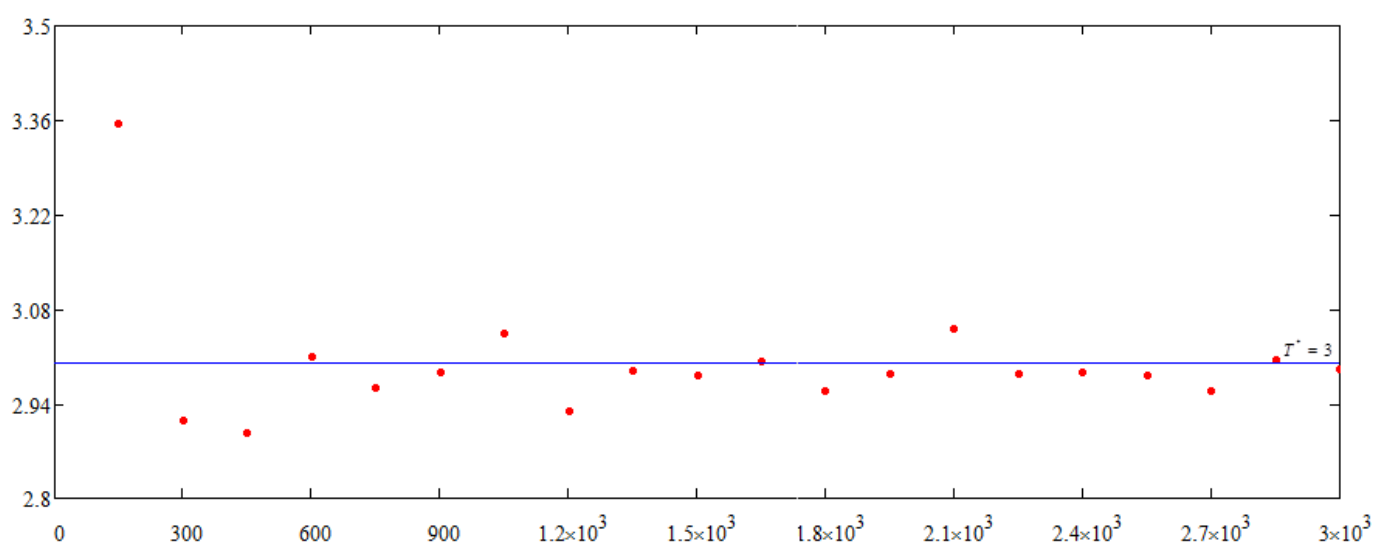

Рис. 4. График зависимости $\hat{M}\left(\hat{T}^{*}\right)$ от $T_{m}$ при $T^{*}=3$

Из анализа результатов табл. 1 и графиков зависимости $\hat{M}\left(\hat{\boldsymbol{T}}^{*}\right)$ от значения $T_{m}$ следует, что стационарный режим функционирования потока устанавливается при $T_{m} \geq 1500$ ед. времени, т.к. выборочное среднее $\hat{M}\left(\hat{\boldsymbol{T}}^{*}\right)$ стремится к постоянному значению.

В табл. 2 приведены результаты для $\operatorname{Var}\left(\hat{\boldsymbol{T}}^{*}\right)$. Структура табл. 2 аналогична структуре табл. 1.

Таблица 2

Численные результаты статистического эксперимента для $\operatorname{Var}\left(\hat{\boldsymbol{T}}^{*}\right)$

\begin{tabular}{|c|c|l|l|l|l|l|l|l|}
\hline $\boldsymbol{T}_{m}$ & & $\mathbf{1 5 0}$ & $\mathbf{3 0 0}$ & $\mathbf{4 5 0}$ & $\mathbf{6 0 0}$ & $\mathbf{7 5 0}$ & $\mathbf{9 0 0}$ & $\mathbf{1 0 5 0}$ \\
\hline \multirow{2}{*}{$\operatorname{Var}\left(\hat{\boldsymbol{T}}^{*}\right)$} & $\boldsymbol{T}^{*}=\mathbf{1}$ & 1.89788 & 0.45199 & 0.43296 & 0.26849 & 0.19075 & 0.20681 & 0.11928 \\
\cline { 2 - 9 } & $\boldsymbol{T}^{*}=\mathbf{3}$ & 2.17312 & 1.51272 & 0.75498 & 0.71807 & 0.62688 & 0.44294 & 0.47239 \\
\hline \multirow{2}{*}{$\boldsymbol{T} \boldsymbol{T}$} & $\mathbf{1 2 0 0}$ & $\mathbf{1 3 5 0}$ & $\mathbf{1 5 0 0}$ & $\mathbf{1 6 5 0}$ & $\mathbf{1 8 0 0}$ & $\mathbf{1 9 5 0}$ & $\mathbf{2 1 0 0}$ & $\mathbf{2 2 5 0}$ \\
\cline { 2 - 9 } & 0.10999 & 0.11771 & 0.08557 & 0.09088 & 0.06747 & 0.07263 & 0.06841 & 0.07537 \\
\cline { 2 - 8 } & 0.28876 & 0.34313 & 0.23482 & 0.2983 & 0.27287 & 0.26733 & 0.23022 & 0.19273 \\
\hline
\end{tabular}

На рис. 4 и 5 приведены графики зависимости выборочной вариации $\operatorname{Var}\left(\hat{\boldsymbol{T}}^{*}\right)$ от времени моделирования $T_{m}$ для $T^{*}=1$ и $T^{*}=3$, построенные по данным табл. 2 .

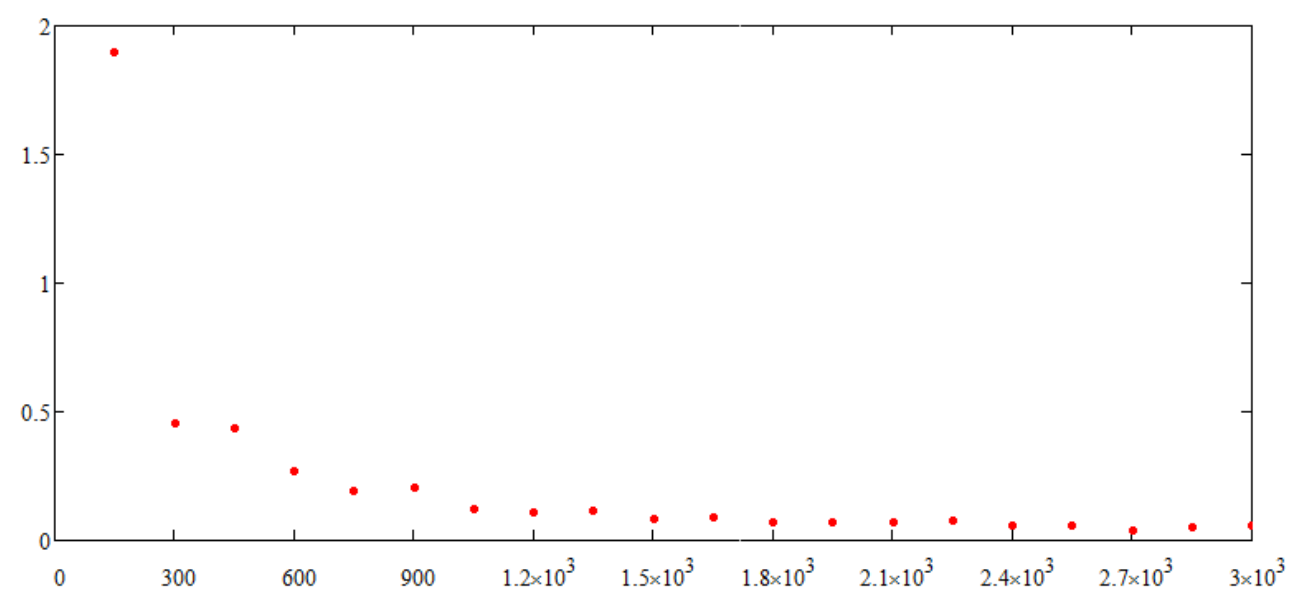

Рис. 5. График зависимости $\operatorname{Var}\left(\hat{\boldsymbol{T}}^{*}\right)$ от $T_{m}$ при $T^{*}=1$ 


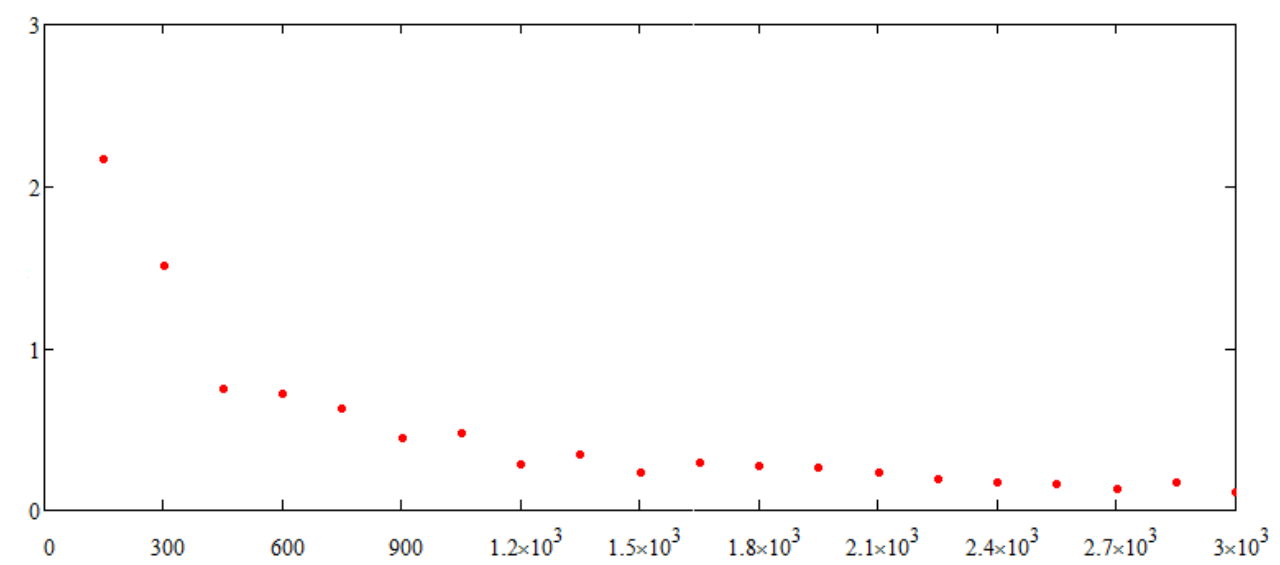

Рис. 6. График зависимости $\operatorname{Var}\left(\hat{\boldsymbol{T}}^{*}\right)$ от $T_{m}$ при $T^{*}=3$

Результаты эксперимента указывают на высокое качество построенной оценки, т.к. выборочная вариация (разброс значений случайной величины $\hat{\boldsymbol{T}}^{*}$ ) стремится к числу, достаточно близкому к нулю, т.е. методика оценивания удовлетворительна и полученную оценку можно принимать за истинную с достаточно малой погрешностью. Также заметим, что выборочная вариация устанавливается возле своего стационарного значения при времени моделирования $T_{m} \geq 1500$ ед. времени. Таким образом, можно считать, что при $T_{m} \geq 1500$ достигается нужная для практики точность.

Подчеркнем, что выборочная вариация при $T^{*}=3$ больше, чем при $T^{*}=1$. Последнее является естественным, т.к. при больших $T^{*}$ происходит большая потеря событий исходного потока, что влечет за собой ухудшение качества оценивания при одинаковых $T_{m}$.

Кроме того, получена состоятельная оценка параметра распределения длительности мертвого времени $T^{*}$, т.к. выполняются условия теоремы о состоятельности оценок [19]: длительности интервалов между моментами наступления событий в потоке являются независимыми случайными величинами; теоретическое среднее существует; решение уравнения моментов существует и единственно.

\section{Заключение}

В данной работе рассмотрен полусинхронный дважды стохастический поток событий с непродлевающимся случайным мертвым временем. Найдены условия рекуррентности потока и исследован альтернирующий рекуррентный полусинхронный дважды стохастический поток событий.

Аналитически получены формулы (5), (6), определяющие плотность вероятности значений длительности интервала между соседними событиями в наблюдаемом рекуррентном потоке при случайном мертвом времени; выведена формула (7) для математического ожидания длительности интервала между соседними событиями.

Методом моментов найдена оценка параметра равномерного распределения длительности случайного мертвого времени, полученная оценка исследована на качество. Приведенные результаты численных расчетов указывают на приемлемое качество оценивания.

\section{ЛИТЕРАТУРА}

1. Cox D.R. The analysis of non-Markovian stochastic processes by the inclusion of supplementary variables // Proceedings of the Cambridge Philosophical Society. - 1955. - V. 51(3). - No. 3. - P. 433-441. 
2. Kingman Y.F. C. On doubly stochastic Poisson process // Proceedings of the Cambridge Philosophical Society. - 1964. - V. 60. - No. 4. - P. 923-930.

3. Бамарин Г.П., Кокотушкин В.А., Наумов В. А. О методе эквивалентных замен расчета фрагментов сетей связи. Ч.1 // Изв. АНССР. Техн. Кибернетика. - 1979. - №6. - С. 92-99. 779.

4. Neuts M.F. A versatile Markovian point process // Journal of Applied Probability. - 1979. - V. 16. - P. 764

5. Lucantoni D.M. New results on the single server queue with a batch markovian arrival process // Communication in Statistics Stochastic Models. - 1991. - V. 7. - P. 1-46.

6. Горцев А.М., Нежельская Л.А. Оценивание длительности мертвого времени и параметров синхронного альтернирующего потока событий // Вестник Томского государственного университета. - 2003. - № S6. - C. 232239.

7. Gortsev A.M., Nezhel'skaya L.A. Estimation of parameters of synchronously alternating Poisson stream of events by the moment method // Telecommunications and Radio Engineering. - 1996. - V. 50. - No. 1. - P. 56-63.

8. Горчев А.М., Нежельская Л.А. Оценивание параметров синхронного дважды стохастического потока событий методом моментов // Вестник Томского государственного университета. - 2002. - № S1-1. - C. 24-29.

9. Nezhel 'Skaya L.A. Probability density function for modulated MAP event flows with unextendible dead time // Communication in Computer and Information Science. - 2015. - V. 564. - P. 141-151.

10. Гориев А.М., Нежельская Л.А. Оценивание параметров асинхронного потока с инициированием лишних событий методом моментов // Вестник Томского государственного университета. - 2006. - № S18. - C. 267273.

11. Леонова М.А., Нежельская Л.А. Вероятность ошибки при оценивании состояний обобщенного асинхронного потока событий // Вестник Томского государственного университета. Управление, вычислительная техника и информатика. - 2012. - № 2 (19). - С. 88-101.

12. Горцев А.М., Зуевич В.Л. Оптимальная оценка состояний асинхронного дважды стохастического потока событий с произвольным числом состояний // Вестник Томского государственного университета. Управление, вычислительная техника и информатика. - 2010. - № 2 (11). С. 44-65.

13. Горцев А.М., Леонова М.А., Нежельская Л.А. Сравнение МП- и ММ-оценок длительности мертвого времени в обобщенном асинхронном потоке событий // Вестник Томского государственного университета. Управление, вычислительная техника и информатика. - 2013. - № 4 (25). - С. 32-42.

14. Калягин А.А., Нежельская Л.А. Сравнение МП- и ММ-оценок длительности мертвого времени в обобщенном полусинхронном потоке событий // Вестник Томского государственного университета. Управление, вычислительная техника и информатика. - 2015. - № 3 (32). - С. 23-32.

15. Гориев А.М., Калягин А.А., Нежельская Л.А. Оценка максимального правдоподобия длительности мертвого времени в обобщенном полусинхронном потоке // Вестник Томского государственного университета Управление, вычислительная техника и информатика. - 2015. - № 1 (30). - С. 27-37.

16. Нежельская Л.А. Оптимальное оценивание состояний полусинхронного потока событий в условиях его частичной наблюдаемости // Вестник Томского государственного университета. - 2000. - № 269. - С. 95-98.

17. Гориев А.М., Калягин А.А., Нежельская Л.А. Совместная плотность вероятностей длительности интервалов обобщенного полусинхронного потока событий при непродлевающемся мертвом времени // Вестник Томского государственного университета. Управление, вычислительная техника и информатика. - 2014. - № 2 (27). C. 19-29.

18. Апанасович B.В., Коляда А.А., Чернявский А.Ф. Статистический анализ случайных потоков в физическом эксперименте. - Минск: Университетское, 1988. - 256 с.

19. Малинковский Ю.В. Теория вероятностей и математическая статистика (часть 2. Математическая статистика). - Гомель: УО «ГГУ им. Ф. Скорины», 2004. - 146 с.

20. Амосов А.А., Дубинский Ю.А., Копченова Н.В. Вычислительные методы для инженеров: учеб. пособие. - М.: Высшая школа, 1994. - 544 с.

DOI: $10.17223 / 978-5-907442-42-9-2021-13$

\title{
ИССЛЕДОВАНИЕ ЭЛЕКТРОМАГНИТНОГО РАССЕЯНИЯ НА ТОНКИХ СООСНЫХ ИДЕАЛЬНО ПРОВОДЯЩЕМ И ДИЭЛЕКТРИЧЕСКОМ ЦИЛИНДРАХ
}

\author{
Дмитренко А.Г., Балашова О.М. \\ Томский государственный университет \\ dmitr.tsu.202@mail.ru, balashovajkz@mail.ru
}

\section{Введение}

Значительный интерес для исследователей представляет изучение рассеяния электромагнитных волн на структурах, состоящих как из идеально проводящих, так и диэлектрических тонких цилиндров конечной длины. Этот интерес обусловлен необходимостью решения таких практически важных проблем, как проблемы радиолокацион- 